\title{
Stress-free-state based structural analysis and construction control theory for staged construction bridges
}

\author{
Shunquan Qin ${ }^{1,2^{*}}$, Kai Wei ${ }^{2}$, Jingxi Qin ${ }^{3}$, Renan Yuan ${ }^{1,2}$, Leiping Xu ${ }^{1}$ and Qilian Dan ${ }^{2}$
}

\author{
* Correspondence: qinshq@cae.cn \\ ${ }^{1}$ China Railway Major Bridge \\ Reconnaissance \& Design Institute \\ Co., Ltd., Wuhan 430050, China \\ ${ }^{2}$ Department of Bridge Engineering, \\ Southwest Jiaotong University, \\ Chengdu 610031, China \\ Full list of author information is \\ available at the end of the article
}

\begin{abstract}
Structural analysis and construction control of staged construction process is a major subject for modern long-span bridges. This paper introduces the concept of stress-freestate variable of structural elements and deduces the mechanical equilibrium equations and geometric shape governing equations for staged construction structures utilizing the minimum potential energy theorem. As the core of stress-free-state theory, the two aforementioned equations demonstrate following principles, 1) when the stress-free-state variable of a structural element is set, the internal force and deformation of the element are unique at the completion state of the structure regardless of its construction process; 2) the stress-free length of a cable is independent of its external loads, change in stress-free length of the cable corresponds to a unique variation of the cable force when load is constant; and 3) the internal force of a structural element can be independent from its geometric shape within the completion state of a staged construction structure through an active manipulation of stress-free-state variables of the element. Stress-free-state theory establishes the stage-to-stage and stage-to-completion relationships for staged construction bridges, provides a direct and efficient method for theoretical calculations and a flexible and convenient approach for the control of staged construction, and makes parallel construction and auto-filtering of thermal and temporary loading effect possible.
\end{abstract}

Keywords: Staged construction bridge, Stress-free-state variable, Stress-free-state theory, Mechanical equilibrium equation, Geometric shape governing equation, Parallel construction

\section{Introduction}

Staged construction, as well as the one-step scaffold supported bridge construction, are two commonly used methods in bridge construction. In the second half of the nineteenth century, the scaffold-free balanced cantilever construction method was first proposed. In the 1930s, the cantilevered staged construction method began to be applied to construct concrete arch bridges. After World War II, the cantilever cast-in-place and the pre-fabricated segmental assembly methods were developed successively benefitting from the development of pre-stressed concrete techniques. (Ge 1986; Qin 2007; Xiang 2001; Li et al. 2009) Represented by the balanced cantilever construction method, the staged

(c) The Author(s). 2020, corrected publication 2021. Open Access This article is licensed under a Creative Commons Attribution 4.0 International License, which permits use, sharing, adaptation, distribution and reproduction in any medium or format, as long as you give appropriate credit to the original author(s) and the source, provide a link to the Creative Commons licence, and indicate if changes were made. The images or other third party material in this article are included in the article's Creative Commons licence, unless indicated otherwise in a credit line to the material. If material is not included in the article's Creative Commons licence and your intended use is not permitted by statutory regulation or exceeds the permitted use, you will need to obtain permission directly from the copyright holder. To view a copy of this licence, visit http://creativecommons.org/licenses/by/4.0/. 
construction has been widely used in the construction of beam bridges, cable-stayed bridges, and arch bridges.

A staged construction bridge undergoes multiple stages of element installations and varying states of the structural system from its project commencement to completion of the bridge construction (Qin 2007). The structural system of the bridge experiences dynamic changes in its construction process as dead loads are being gradually applied to the structure during the staged construction process. In essence, staged construction is a complicated time-dependent process. Furthermore, the construction of the bridge needs to satisfy the designed internal force and geometric shape at its completion state, while maintaining structural safety during the process. These requirements bring a great deal of complexity to the structural analysis and construction control of staged construction bridges.

The basic goal of the structural analysis and control of staged bridge construction is to satisfy the designed internal force and geometric shape at the completion state, while maintaining structural safety during the construction process (Qin 2007). Since the structural system states and the dead load applications of staged bridge construction are time-dependent, the traditional structural analysis theories that do not consider the effect of the construction process cannot be implemented directly (Oden and Ripperger 1986). Therefore, scholars have made efforts to take the construction stages of the bridge into consideration and proposed several methods based on the incremental structural analysis of each construction stage, such as the forward analysis method and the backward analysis method (Ge 2003; Qin 2007; Xiang 2001). Among these methods, the backward analysis method is most widely adopted. The backward analysis starts from the completion state of the bridge and proceeds with its structural analysis stage by stage backwards to the initial state of the bridge. The method calculates the internal force and deformation of each construction stage to serve as the reference for construction operations. However, these incremental structural analysis methods generally have three disadvantages, including 1) the computational complexity from modified construction process, 2) the inability to consider temperature inconsistency between the design and construction site, and 3) the inability to accommodate parallel construction operations when temporary construction loads are present (Qin 2007; Qin 2008a).

Stress-free-state theory for structural analysis and construction control of staged construction bridge started to take shape in the late 1980s (Qin 2007). In 1992, Qin et al. published a paper and proposed the completion state shape approximation of a staged construction bridge by controlling the stress-free-state variables of structural elements at the first time (Qin and Lin 1992). In 2003, Qin proposed the parallel construction operation and automatic filtering technique of temporary load effects by applying the stress-free-state theory. In 2008, Qin derived the mechanical equilibrium equation of staged construction structures with energy method by introducing the stress-free-state variables of structural elements, thus further explained the relationship between the internal force and deformation of completion state of staged construction structures and their formation processes (Qin 2008b). The theoretical development and engineering applications of the stress-free-state theory were then studied extensively. Qin et al. established the mechanical equilibrium equation for staged construction spatial frame structures (Qin et al. 2013). Xu et al. developed the mechanical equilibrium equation for plate and shell element and Timoshenko beam element (Xu 2016; Xu et al. 2013; 
$\mathrm{Xu}$ et al. 2015). Yuan et al. derived the mechanical equilibrium equation for planar link element and planar beam element including geometric nonlinearity (Yuan et al. 2014a; Yuan et al. 2014b). Dan et al. (2017a) developed the geometric shape governing equation for staged construction structures (Dan 2017; Dan et al. 2017a). In 1992, the first successful application of the stress-free-state theory was in the staged construction control of the Second Yangtze River Bridge in Wuhan (Qin et al. 1995), thereafter, it has been used in the construction control of up to one hundred long-span bridges and all archived great successes (Huang 2003; Huang 2000; Gao and Huang 2003; Wu 2006; Liu and Wen 2003; Shi et al. 2010; Sun et al. 2011; Wu et al. 2011; Wu et al. 2012; Wan 2012; Yuan and Qin 2012; Li et al. 2013; Yue et al. 2013; Que et al. 2013; Tan and Yu 2013; Yan et al. 2013; Zhang et al. 2015; Dan et al. 2017b). Extensive theoretical studies and numerous engineering applications show that the stress-free-state theory is not only especially advantageous in the staged analysis and construction control of cable-stayed bridges, but also considered efficient for the bridges with any other structural types and materials (Qin 2007).

Structural analysis and construction control of staged construction process is a major subject for modern long-span bridges. This paper introduces the concept of stress-freestate variables of structural elements and deduces the mechanical equilibrium equations and geometric shape governing equations for staged construction structures utilizing the minimum potential energy theorem. The basic principles of the stress-free-state theory are then proposed on the basis of above equations. Finally, the applications of the stress-free-state theory in the practical structural analysis and construction control of staged construction bridges are summarized and discussed.

\section{Mechanical equilibrium equation and geometric shape governing equation of the staged construction structure}

\subsection{Definition of stress-free-state variable}

From the perspective of the structural element, the stress-free-state variable is defined as the geometric shape of the element, whose stress due to external loads is absent (Qin 2007; Qin and Lin 1992; Qin 2008b). In the finite element modeling, the element must be meshed to a very refined level, where the effect of the initial stress on the overall structural behavior can be neglected. In the construction of bridges, the element refers to the structural element that is installed separately.

According to above definition, the stress-free-state variables for a planar beam element include stress-free length and stress-free curvature (Qin 2007), while for a spatial beam element, its stress-free rotation angle should also be included. (Qin et al. 2013) The stress-free-state variables for a shell element include: stress-free curvature, stressfree rotation curvature, and its stress-free shape described in plane stress (Xu 2016; Xu et al. 2013).

\subsection{Mechanical equilibrium equation of staged construction structures}

Forward method, backward method, and other similar analysis methods for staged construction of structures are derived on the basis of the mechanical equilibrium equation of one-step construction of structures (Qin 2007; Oden and Ripperger 1986): 
where $\mathrm{K}$ is the structural stiffness matrix, $\delta$ is the structural deformation and $\mathrm{P}$ is the external loading vector. The internal force and deformation of the structure at its completion state can be obtained by calculating and adding up the incremental variables for each step of staged construction. These incremental structural analysis methods generally have three disadvantages, including: 1) the computational complexity from modified construction process, 2) the inability to consider temperature inconsistency between the design and construction site, and 3) the inability to accommodate parallel construction operations when temporary construction loads are present (Qin 2007; Qin 2008a).

In order to overcome above limitations, with the addition of the stress-free-state variables, the derivations of mechanical equilibrium equations for different types of elements neglecting the interim states of the structures utilizing the minimum potential energy theorem are briefly summarized.

\subsubsection{Planar beam element}

As shown in Fig. 1, the structure is a cable-stayed bridge, which is completed by a multistaged construction with any arbitrary construction process. $P_{x i} P_{y i}$ and $M_{i}(i=1,2, \ldots, \mathrm{k})$ is the axial force, shear force and moment at the node $i$, respectively. The element shown in Fig. 2 is an arbitrary element of the structure in Fig. 1, its nodal displacement in completion state is $\left(\bar{u}_{i}, \bar{v}_{i}, \bar{\theta}_{i}\right),(\mathrm{i}=1,2,3 \ldots \mathrm{m}), l_{0}$ is the stress-free length of the element, $l$ is the calculation length in finite element model, and the stress-free curvature at the two nodes are $\kappa_{i 0}$ and $\kappa_{j 0}$, respectively. Variation of the element length in its completion state can be written as:

$$
\Delta l=\bar{u}_{j}-\bar{u}_{i}+l-l_{0}
$$

Variation of the curvatures at two nodes of the element in its completion state can be expressed respectively as:

$$
\begin{aligned}
& \Delta \kappa_{i}=\frac{2}{l^{2}}\left(3 \bar{v}_{j}-3 \bar{v}_{i}-2 l \bar{\theta}_{i}-l \bar{\theta}_{j}\right)-\Delta \kappa_{i 0} \\
& \Delta \kappa_{j}=\frac{2}{l^{2}}\left(3 \bar{v}_{i}-3 \bar{v}_{j}+l \bar{\theta}_{i}+2 l \bar{\theta}_{j}\right)-\Delta \kappa_{j 0}
\end{aligned}
$$

No matter what the construction process is, the strain energy of the element in its completion state only depends on its deformation that actually occurs (Oden and

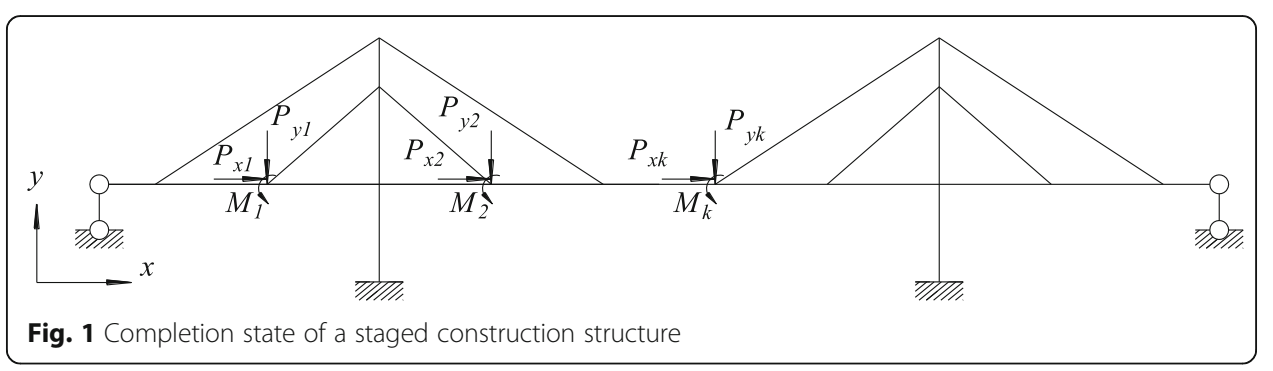




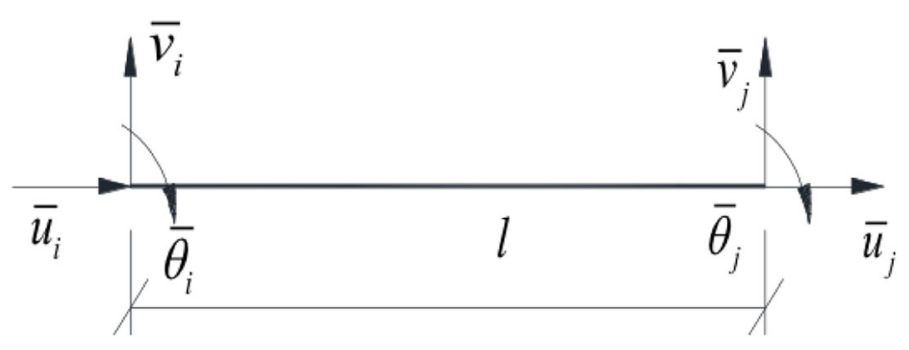

Fig. 2 Element in local coordinates system

Ripperger 1986; Qin 2008b). Therefore, the strain energy of the element along axial and curvature direction can be expressed as:

$$
\begin{aligned}
& U_{N}=\frac{E A}{2 l} \cdot \Delta l^{2}=\frac{E A}{2 l}\left[\bar{u}_{j}-\bar{u}_{i}+\left(l-l_{0}\right)\right]^{2} \\
& U_{M}=\frac{E I}{2} \int_{0}^{l} \Delta \kappa^{2}(x) d x=\frac{E I l}{6}\left(\Delta \kappa_{i}{ }^{2}+\Delta \kappa_{i} \Delta \kappa_{j}+\Delta \kappa_{j}{ }^{2}\right)
\end{aligned}
$$

where $E, A$ and $I$ is Young's modulus, area and moment of inertia for the element, respectively. Since the completion state of the structure shown in Fig. 1 is in equilibrium, the variation of the strain energy equals (Oden and Ripperger 1986)

$$
\delta \Pi=0
$$

The following mechanical equilibrium equation of staged construction structure can then be obtained (Qin 2008b),

$$
\mathbf{K} \boldsymbol{\delta}=\mathbf{P}+\mathbf{P}_{0}
$$

where the first 3 terms of Eq. 8 are identical to that of Eq. 1 . The fourth term $P_{0}$ is the generalized load, which can be written as:

$$
\begin{aligned}
& \mathbf{P}_{0}=\sum_{i=1}^{n} \mathbf{P}_{0 i}^{e} \\
& \mathbf{P}_{0 i}^{e}=\left\{\begin{array}{c}
\left(l-l_{0}\right) \frac{E A}{l} c+\left(\kappa_{i 0}-\kappa_{j 0}\right) \frac{E I}{l} s \\
\left(l-l_{0}\right) \frac{E A}{l} s-\left(\kappa_{i 0}-\kappa_{j 0}\right) \frac{E I}{l} c \\
-\left(l-l_{0}\right) \frac{E A}{l} c-\left(\kappa_{i 0}-\kappa_{j 0}\right) \frac{E I}{l} s \\
-\left(l-l_{0}\right) \frac{E A}{l} s+\left(\kappa_{i 0}-\kappa_{j 0}\right) \frac{E I}{l} c \\
\kappa_{j 0} \cdot E I
\end{array}\right\}
\end{aligned}
$$

where $c$ and $s$ is the cosine function and sine function of the angle between the local coordinate system and the global coordinate system. $\mathrm{P}_{0}$ is the generalized load and a function of stress-free-state variable. Equation 8 is the mechanical equilibrium equation for staged construction structures, which describes the structural equilibrium condition for staged construction structure in its completion state. Equation 9 and 10 reveal that, Eq. 8 becomes identical to Eq. 1 , when $l=l_{0}, \kappa_{i 0}=$ $\kappa_{j 0}=0$ and $\mathrm{P}_{0}=0$. 


\subsubsection{Spatial beam element}

The mechanical equilibrium equation for staged construction structure of a spatial beam element (shown in Fig. 3) can also be derived and has the same format as Eq. 8. The $\boldsymbol{P}_{0 i}^{e}$ term is given as follows (Qin et al. 2013),

$$
\mathbf{P}_{0 i}^{e}=\left\{\begin{array}{c}
\frac{E A c_{11}}{l}\left(l-l_{0}\right)-\frac{E I c_{21}}{l}\left(\kappa_{z i 0}-\kappa_{z j 0}\right)-\frac{E I c_{31}}{l}\left(\kappa_{y i 0}-\kappa_{y j 0}\right) \\
\frac{E A c_{12}}{l}\left(l-l_{0}\right)-\frac{E I c_{22}}{l}\left(\kappa_{z i 0}-\kappa_{z j 0}\right)-\frac{E I c_{32}}{l}\left(\kappa_{y i 0}-\kappa_{y j 0}\right) \\
\frac{E A c_{13}}{l}\left(l-l_{0}\right)-\frac{E I c_{23}}{l}\left(\kappa_{z i 0}-\kappa_{z j 0}\right)-\frac{E I c_{33}}{l}\left(\kappa_{y i 0}-\kappa_{y j 0}\right) \\
-E I c_{31} \kappa_{z i 0}-E I c_{21} \kappa_{y i 0}-\frac{G I_{p} c_{11}}{l} \phi_{0} \\
-E I c_{32} \kappa_{z i 0}-E I c_{22} \kappa_{y i 0}-\frac{G I_{p} c_{12}}{l} \phi_{0} \\
-E I c_{33} \kappa_{z i 0}-E I c_{23} \kappa_{y i 0}-\frac{G I_{p} c_{13}}{l} \phi_{0} \\
-\frac{E A c_{11}}{l}\left(l-l_{0}\right)+\frac{E I c_{21}}{l}\left(\kappa_{z i 0}-\kappa_{z j 0}\right)+\frac{E I c_{31}}{l}\left(\kappa_{y i 0}-\kappa_{y j 0}\right) \\
-\frac{E A c_{12}}{l}\left(l-l_{0}\right)+\frac{E I c_{22}}{l}\left(\kappa_{z i 0}-\kappa_{z j 0}\right)+\frac{E I c_{32}}{l}\left(\kappa_{y i 0}-\kappa_{y j 0}\right) \\
-\frac{E A c_{13}}{l}\left(l-l_{0}\right)+\frac{E I c_{23}}{l}\left(\kappa_{z i 0}-\kappa_{z j 0}\right)+\frac{E I c_{33}}{l}\left(\kappa_{y i 0}-\kappa_{y j 0}\right) \\
E I c_{31} \kappa_{z j 0}+E I c_{21} \kappa_{y j 0}+\frac{G I_{p} c_{11}}{l} \phi_{0} \\
E I c_{32} \kappa_{z j 0}+E I c_{22} \kappa_{y j 0}+\frac{G I_{p} c_{12}}{l} \phi_{0} \\
E I c_{33} \kappa_{z j 0}+E I c_{23} \kappa_{y j 0}+\frac{G I_{p} c_{13}}{l} \phi_{0}
\end{array}\right\}
$$

where $\phi_{0}$ denotes the stress-free rotation angle of the element, and $c_{i j}, i=1 \sim 3, j=1 \sim 3$, represents the cosine function of the angle between the local coordinate system and the global coordinate system.

\subsubsection{Plate and shell element}

The mechanical equilibrium equation for staged construction structures for plate and shell element (illustrated in Fig. 4) also has the same format as Eq. 8. The $\mathbf{P}_{0}$ term is shown as follows (Xu 2016; Xu et al. 2013),

$$
\mathbf{P}_{0 i}^{e}=\int_{-1}^{1} \int_{-1}^{1} \mathbf{B}^{\mathrm{T}} \mathbf{D}_{\mathrm{p}} \mathbf{A}_{\mathrm{p}} \boldsymbol{\varepsilon}_{\mathrm{g} 0} \operatorname{det}\left(\mathbf{J}_{\mathrm{p}}\right) d \xi d \eta+\int_{-1}^{1} \int_{-1}^{1} \mathbf{B}^{\mathrm{T}} \mathbf{D}_{\mathrm{b}} \mathbf{A}_{\mathrm{b}} \mathbf{k}_{\mathrm{g} 0} \operatorname{det}\left(\mathbf{J}_{\mathrm{b}}\right) d \xi d \eta
$$

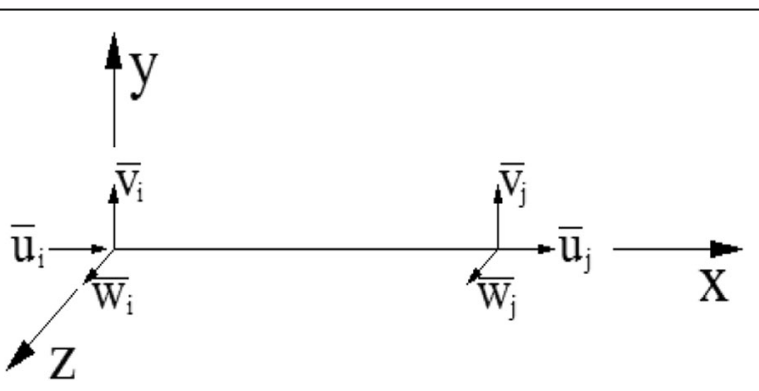

Fig. 3 Three dimensional beam element 


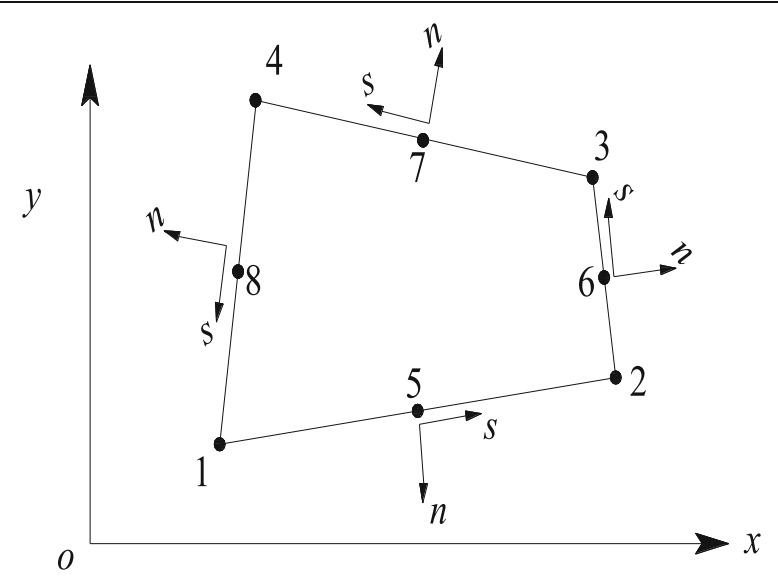

Fig. 4 Shell element

where $\boldsymbol{B}$ is the strain matrix of the element, $\boldsymbol{\varepsilon}_{\mathrm{g} 0}$ and $\boldsymbol{\kappa}_{\mathrm{g} 0}$ denotes the stress-free axial and bending deformation vectors at the Gaussian integration point g, respectively.

\subsubsection{Planar beam element including geometric nonlinearity}

As shown in Fig. 5, when the geometric nonlinearity is included in planar beam element, taken the stress-free-state of the structural element as the starting point in the calculation of the total potential energy of structural system, the mechanical equilibrium equation can be derived by minimum potential energy theorem as (Yuan et al. 2014b),

$$
\sum_{i=1}^{m} \mathbf{T}^{\mathbf{T}}\left(\overline{\mathbf{k}}^{e}+\overline{\mathbf{k}}_{\varepsilon 0}^{e}\right) \mathbf{T} \boldsymbol{\delta}=\mathbf{K} \boldsymbol{\delta}=\mathbf{P}+\sum_{\mathbf{i}=\mathbf{1}}^{\mathbf{m}} \mathbf{P}_{0}^{e}
$$

where

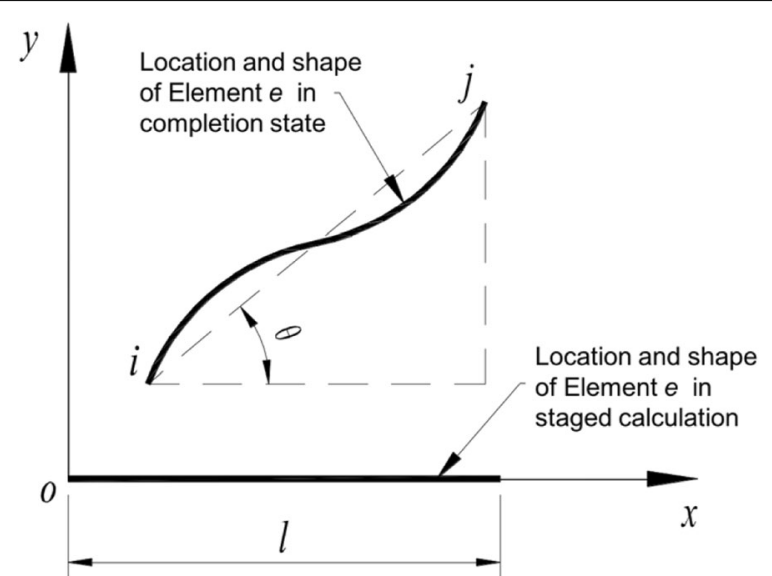

Fig. 5 Nonlinear plane beam element 


$$
\begin{aligned}
& \overline{\mathbf{k}}_{\varepsilon 0}^{e}=\frac{E A\left(l-l_{0}\right)}{l}\left.\begin{array}{cccccc}
\frac{1}{l} & 0 & 0 & -\frac{1}{l} & 0 & 0 \\
0 & \frac{6}{5 l} & \frac{1}{10} & 0 & -\frac{6}{5 l} & \frac{1}{10} \\
0 & \frac{1}{10} & \frac{2 l}{15} & 0 & -\frac{1}{10} & -\frac{l}{30} \\
-\frac{1}{l} & 0 & 0 & \frac{1}{l} & 0 & 0 \\
0 & -\frac{6}{5 l} & -\frac{1}{10} & 0 & \frac{6}{5 l} & -\frac{1}{10} \\
0 & \frac{1}{10} & -\frac{l}{30} & 0 & -\frac{1}{10} & \frac{2 l}{15}
\end{array}\right] \\
& \mathbf{P}_{0}^{e}=\left\{\begin{array}{c}
\left(l-l_{0}\right) \frac{E A}{l} c+\left(\kappa_{i 0}-\kappa_{j 0}\right) \frac{E I}{l} s \\
\left(l-l_{0}\right) \frac{E A}{l} s-\left(\kappa_{i 0}-\kappa_{j 0}\right) \frac{E I}{l} c \\
-\left(l-l_{0}\right) \frac{E A}{l} c-\left(\kappa_{i 0}-\kappa_{j 0}\right) \frac{E I}{l} s \\
-\left(l-l_{0}\right) \frac{E A}{l} s+\left(\kappa_{i 0}-\kappa_{j 0}\right) \frac{E I}{l} c \\
\kappa_{j 0} E I
\end{array}\right\}
\end{aligned}
$$

\subsection{Geometric shape governing equation of staged construction structure}

For staged construction of bridges, the completion geometric shape of a bridge is as important as the internal force (Yu and Li 2012). The so-called "geometric shape of a bridge" is defined as the spatial location of the structural characteristic points of a completed bridge under dead loads.

A common technique used in practice to modify completion spatial location (geometric shape) of a structure is to alter the initial shapes and the respective geometric relations of structural elements, as shown in Fig. 6. In essence, the initial shapes and respective geometric relations of structural elements are determined by the stress-free length and curvature of the elements. Dan (2017) derived the relationship between bridge segment's geometry and its elemental stress-free-state variables (Eq. 16),

$$
\left\{\begin{array}{l}
\theta_{i 0}=-\frac{l}{6}\left(2 \kappa_{i 0}+\kappa_{j 0}\right) \\
\theta_{j 0}=\frac{l}{6}\left(\kappa_{i 0}+2 \kappa_{j 0}\right)
\end{array}\right.
$$

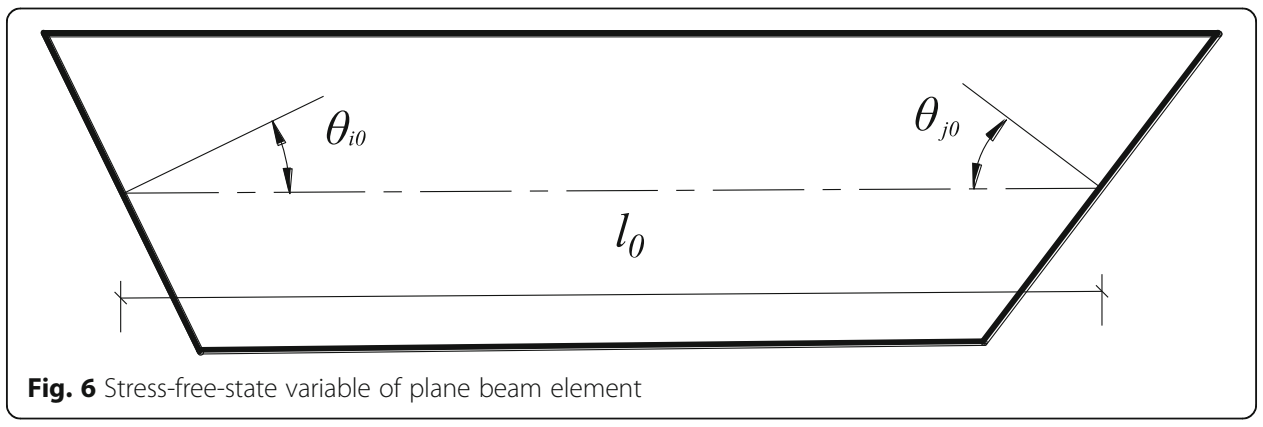


Controlling the stress-free-state variable of the element actively can archive different geometric shape. By setting stress-free-state variable of an element as the unknown value and establishing its relationship with geometric shape at the completion state of a structure, linear function of staged construction of structures can be derived.

As shown in Fig. 1, the structure is constructed by a multi-stage process and takes only dead loads. There are $\mathrm{n}$ elements and $\mathrm{m}$ nodes on the structure. The total load applied on the structure is $\mathbf{P}_{k}\left(P_{x_{k}}, P_{y_{k}}, M_{k}\right)(k=1,2, \cdots, r)$, and the geometric shape is described in a fixed coordinates system with rotation as $\mathbf{C}_{\lambda}\left(x_{\lambda}, y_{\lambda}, \theta_{\lambda}\right)(\lambda=1,2, \cdots, m)$.

The axial and bending strain energy of an element can be expressed as a function of its coordinates and rotation. Since the work potential on the element is also a function of its coordinates, the total potential energy can be expressed parametrically:

$$
\Pi=\Pi\left(x_{\lambda}, y_{\lambda}, \theta_{\lambda}\right)(\lambda=1,2, \cdots, m)
$$

Because the structure in its completion is in equilibrium, the principle of stationary potential energy holds and we can have

$$
\frac{\partial \boldsymbol{\Pi}}{\partial \mathbf{C}_{y}}=\frac{\partial \sum_{e=1}^{n} \boldsymbol{U}}{\partial \mathbf{C}_{y}}+\frac{\partial \mathbf{W}}{\partial \mathbf{C}_{y}}=0 \quad(\lambda=1,2,3 \cdots, m)
$$

thus,

$$
\left(\sum_{e=1}^{n} \mathbf{L}^{\mathbf{e}}\right) \mathbf{\kappa}=\left(\sum_{e=1}^{n} \mathbf{k}^{\mathbf{e}}\right) \mathbf{C}-\mathbf{P}
$$

where $P$ is the external load vector, $\boldsymbol{k}^{e}$ is the structural stiffness matrix, $\boldsymbol{\kappa}$ is the stressfree-state variable column vector,

$$
\mathbf{\kappa}=\left\{\begin{array}{llllllllll}
l_{01} & \kappa_{i 01} & \kappa_{j 01} & l_{02} & \kappa_{i 02} & \kappa_{j 02} & \cdots & l_{0 n} & \kappa_{i 0 n} & \kappa_{j 0 n}
\end{array}\right\}
$$

where $\sum^{n} \mathbf{L}^{\mathbf{e}}$ is the structural geometric shape governing matrix, consisted of element geometric shape governing matrix.

$$
\mathbf{L}^{e}=\left[\begin{array}{ccc}
-\frac{E A}{l} & 0 & 0 \\
0 & -\frac{E I}{l} & \frac{E I}{l} \\
0 & -E I & 0 \\
\frac{E A}{l} & 0 & 0 \\
0 & \frac{E I}{l} & -\frac{E I}{l} \\
0 & 0 & E I
\end{array}\right]
$$

The assembling rule of structural geometric shape governing matrix is to arrange its row position according to the node degrees of freedom contained in the element, and to arrange its column position according to the element numbers and the element stressfree-state variable numbers. Therefore, the total number of equations in a geometric shape governing equation equals to the total number of structural degrees of freedom, the number of unknown variables equals to the total number of stress-free-state variables. 
Equation 19 is geometric shape governing equation for staged construction structures. When the node coordinates $\mathbf{C}$ is given for the completion state of the structure, the stress-free-state variable of all elements of the structure can be obtained by solving structural geometric shape governing equation.

For structurally determinant structures, with three additional boundary conditions that limits rigid body displacement, Eq. 19 can be solved directly. For structurally indeterminant structures, supplemental internal load equations for redundant structural members and reaction forces for redundant external constraints are required to make geometric shape governing equation solvable. Internal load equations for redundant structural members are shown as follows (Dan 2017; Dan et al. 2017a),

$$
\begin{aligned}
& l_{0}=\left(x_{j}-x_{i}\right) c+\left(y_{j}-y_{i}\right) s-\frac{F_{N} l}{E A} \\
& \kappa_{i 0}=\frac{2}{l^{2}}\left[3\left(y_{j}-y_{i}\right) c-3\left(x_{j}-x_{i}\right) s-2 l \theta_{i}-l \theta_{j}\right]-\frac{M_{i}}{E I} \\
& \kappa_{j 0}=\frac{2}{l^{2}}\left[-3\left(y_{j}-y_{i}\right) c+3\left(x_{j}-x_{i}\right) s+l \theta_{i}+2 l \theta_{j}\right]-\frac{M_{j}}{E I}
\end{aligned}
$$

The additional conditions for structurally indeterminant geometric shape governing equation serve as the necessary supplement for solution of equation. These additional conditions also guarantee consistency of internal loads between completion states and the designs for the structures.

\section{Basic principles of stress-free-state theory}

Principle 1: when the stress-free-state variable of a structural element is set, the internal force and deformation of the element are unique at the completion state of the structure regardless of its construction process (Qin 2007; Qin 2003; Qin 2008b).

According to the mechanical equilibrium equation for staged construction structures (Eq. 8), the construction process are not specified. In other words, as long as the elemental stress-free-state variables are set, the internal force and displacement of a staged construction structure at its completion state are unique even when it is constructed with different processes. Therefore, for a staged construction structure with given external loads, structural stiffness, and elemental stress-free-state variable, its corresponding internal loads and deformation is unique and independent of stages and construction process (Qin 2007; Qin 2003; Qin 2008b).

However, in bridge construction practice, for structures with the same external load but built with different construction processes, it is common to see some differences between completion state internal loads and displacements, which seemingly suggest that internal loads and displacements are related to construction processes. This can be explained by the fact that different processes lead to the inconsistency of the stressfree-state length and curvature of the structural elements, which in turn produces different internal loads and displacements at the completion states of the structures.

Therefore, as long as the stress-free-state of the element in the completion state is determined, the completion internal force and displacement of the element become independent from the construction process. Thus, the complex control problem of staged construction structure process can be simplified into a simple problem of controlling the stress-free-state of structural elements. Understanding the substance related to the 
process will be of great assistance to structural analysis and construction control of the staged construction structures, especially for concrete bridges. Deformation due to creep and shrinkage in reinforced concrete bridge can hence be taken as the changes of stress-free length and curvature of the structural elements.

Principle 2: the stress-free length of the cable is independent of its external loads, and the change in stress-free length corresponds to a unique variation of the cable force when load is constant (Qin 2007; Qin 2003; Qin 2008b).

Taken two construction stages shown in Fig. 7 as an example, assume there is only changes in the external load between stage 1 and stage 2 , the cable force increment can be calculated with the following mechanical equilibrium equation of one-step construction structure:

$$
\mathbf{K}\left(\boldsymbol{\delta}_{1}-\boldsymbol{\delta}_{2}\right)=\mathbf{P}_{1}-\mathbf{P}_{2}
$$

According to the mechanical equilibrium equation of the staged construction structure, there is:

Stage 1:

$$
\mathbf{K} \boldsymbol{\delta}_{1}=\mathbf{P}_{1}+\mathbf{P}_{01}
$$

Stage 2:

$$
\mathbf{K} \boldsymbol{\delta}_{2}=\mathbf{P}_{2}+\mathbf{P}_{02}
$$

Subtracting Eq. 27 from Eq. 26 yields:

$$
\mathbf{K}\left(\boldsymbol{\delta}_{1}-\boldsymbol{\delta}_{2}\right)=\left(\mathbf{P}_{1}-\mathbf{P}_{2}\right)+\left(\mathbf{P}_{01}-\mathbf{P}_{02}\right)
$$

Substituting Eq. 25 into Eq. 27:

$$
\mathbf{P}_{01}=\mathbf{P}_{02}
$$

It is obvious that the stress-free-state variable of the cable element is an inherent property and does not change with the external loading. To change stress-free length of a cable element, the alterations to physical properties such as geometric shape needs

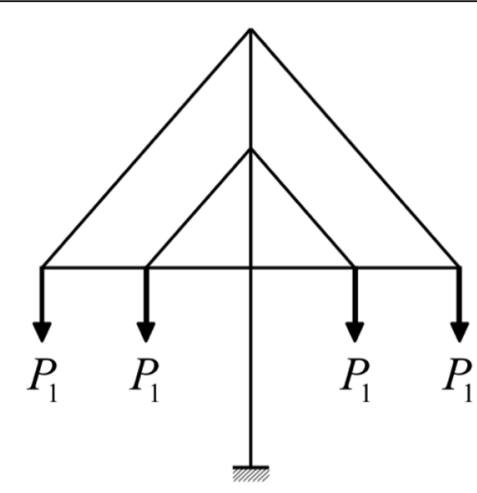

a) Stage 1

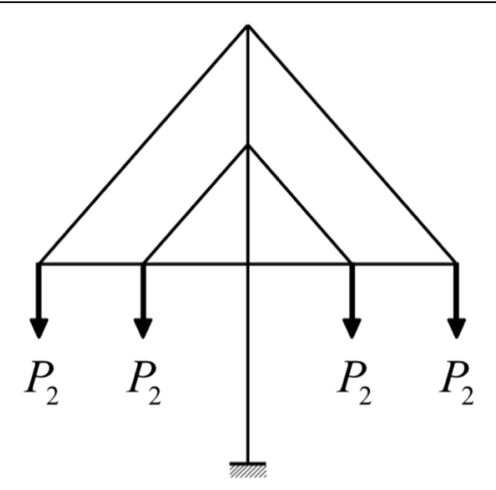

b) Stage 2

Fig. 7 Construction process. a Stage 1. b Stage 2 
to be made. In cable stayed bridges, change in stress-free length of a stay cable is performed by manually modifying cable-end screws location using a hydraulic jack.

When the external loads remain constant, $\mathbf{P}_{1}-\mathbf{P}_{2}=0$, Eq. 28 becomes

$$
\mathbf{K}\left(\boldsymbol{\delta}_{1}-\boldsymbol{\delta}_{2}\right)=\mathbf{P}_{01}-\mathbf{P}_{02}
$$

Equation 30 describes how the change of stress-free-state variable affects the structural internal force and geometric shape of cables, and it also indicates that when modification is made to stress-free length of the cable, the modification corresponds to a unique change in structural internal loads.

Principle 3: The internal force of a structural element can be independent from its geometric shape within the completion state of a staged construction structure through an active manipulation of stress-free-state variable of the element.

According to Eq. 19, when the completion state of a staged construction bridge is structurally determinant, the internal loads of the structure are only subjected to conformation of nodal force equilibrium while the deformation conformation is not required. Stressfree-state variables of structural elements for a bridge at its completion state can be solved by the geometric shape governing equation separately, therefore the internal force and geometric shape are mutually independent at the completion state of the bridge.

When the completion state of a staged construction bridge is structurally indeterminant, with additional internal load equations for redundant members and reaction forces for redundant external constraints, the elemental stress-free-state variables that satisfy completion state of the bridge can be obtained. These supplemental conditions, in essence, guarantee the conformation of internal loads at completion state with the actual design of the structure. It is clear that the internal loads and geometric shapes are mutually independent at completion state of the structure.

\section{Application examples of stress-free-state theory in engineering practice}

\subsection{Structural analysis of staged construction process}

Shown in Fig. 8 is the staged construction of a cable stayed bridge. The key in staged construction bridge installation calculation is to find the internal loads and displacement of the interim stage (a) through (e) that satisfy the internal loads and displacement at its completion stage.

According to stress-free-state theory principle 1, as long as stress-free-state variables of structural elements are provided during structural analysis, the internal loads and displacement are independent of interim stages. Calculation for internal loads and displacement of the interim stage (c) does not require backwards analysis from interim stages (f), (e), and (d). It only requires the stress-free-state variables of installed structural elements

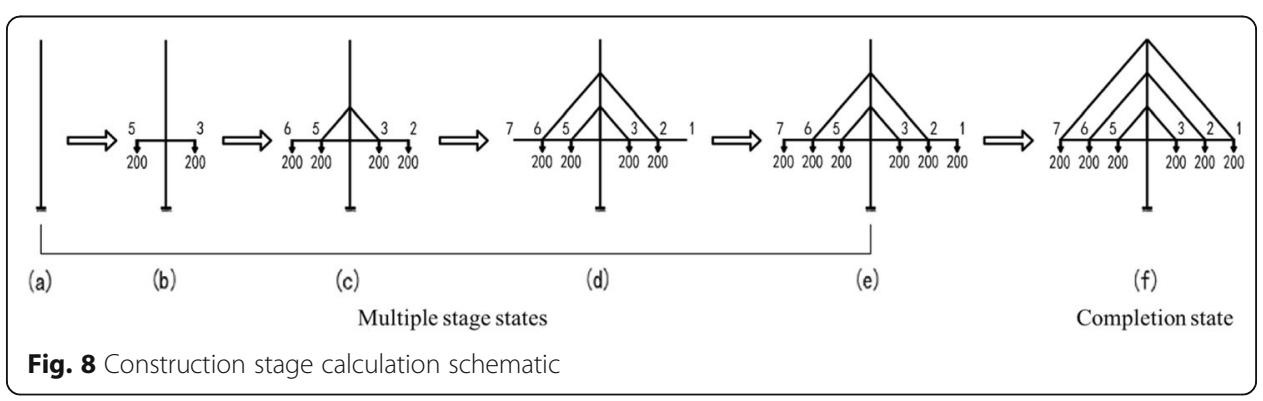


at the stage (c) to be equal to that of the same elements at the completion state of the structure, then the internal loads and displacement can be directly obtained by solving staged structure mechanical equilibrium (Eq. 8), given that external loading used for the analysis only includes all the loadings applied to the structure at the stage (c). The same procedure can be used to solve internal loads and displacements for interim stages (e), (d), (b), and (a).

If the internal loads of interim stages directly obtained from eq. 8 do not satisfy construction safety criteria, it indicates that the stress-free length of the cable could not be stretched into the desired shape in one step. Utilizing the relationship between cable length modification and cable load (Eq. 30) from principle 2 of stress-free-state theory, stress-free length of the cable can be stretched into the desired shape in separate steps (Qin 2007; Qin 2009; Qin 2008c).

\subsection{Parallel construction operation of cable supported bridges}

As shown in Fig. 9, Stage a and Stage b are two construction stages during a staged construction of a cable stayed bridge. Between stage a and stage $\mathrm{b}$, in addition to the cable length modification, there is also a loading increment equal to $P_{2}-P_{1}$.

In stage a, the internal force matrix of the cables is $T=\left\{T_{1}, T_{2}, T_{3}, T_{4}\right\}$ with $P_{1}$ loading. Two parallel construction operations are performed: length modification of cables $C_{1} \sim C_{4}$ (modifying a location of cable end screws with a hydraulic jack) and loading increment $P_{2}-P_{1}$ (caused by construction load change). In stage $\mathrm{b}$, cable loadings become $\boldsymbol{T}^{\prime}=\left\{T_{1}{ }^{\prime}, T_{2}{ }^{\prime}, T_{3}{ }^{\prime}, T_{4}{ }^{\prime}\right\}$. Traditionally, since construction loading change leads to change in cable loadings, cable load modification operation shall not be parallelly carried out with other operations that might cause loading change. Therefore, research on method that allows parallel operation is necessary.

According to mechanical equilibrium equation of staged construction structure,

Stage a:

$$
\mathbf{K} \boldsymbol{\delta}_{1}=\mathbf{P}_{1}+\mathbf{P}_{01}
$$

Stage b:

$$
\mathbf{K} \boldsymbol{\delta}_{2}=\mathbf{P}_{2}+\mathbf{P}_{02}
$$

Subtract Eq. 31 from Eq. 32,
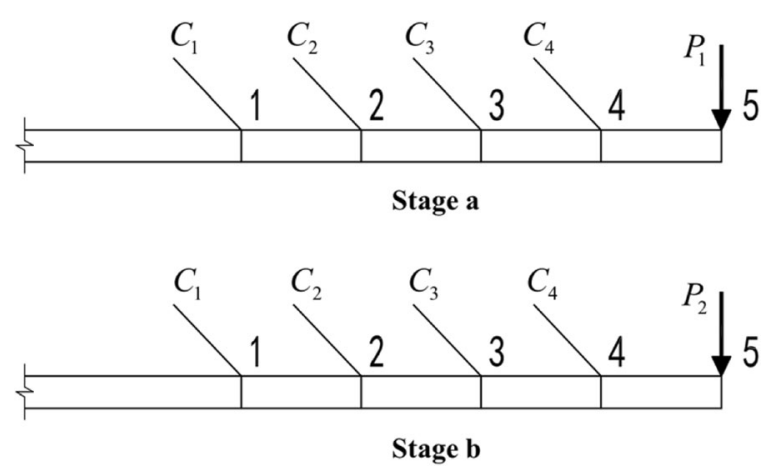

Fig. 9 Staged construction process for cable stayed bridge 


$$
\mathbf{K}\left(\boldsymbol{\delta}_{2}-\boldsymbol{\delta}_{1}\right)=\left(\mathbf{P}_{2}-\mathbf{P}_{1}\right)+\left(\mathbf{P}_{02}-\mathbf{P}_{01}\right)
$$

Equation 33 reveals that changes in internal force and displacement between stage $b$ and stage a only correspond to loading changes and changes in stress-free length of the cable and are independent of sequence of construction operations such as cable load modification.

Therefore, it is possible to carry out parallel construction operations by modifying stress-free length (by modifying location of cable end screws) instead of cable load. The sequence of modifying cables $C_{1} \sim C_{4}$ can also be conducted arbitrarily (Qin 2003; Qin 2008d).

\subsection{Other engineering applications}

Further applications are possible in staged bridge construction by implementing the staged construction structural mechanical equilibrium equation, geometric shape governing equation, and the three principles of stress-free-state theory. Improved structural construction analysis and control can be achieved by considering the effect of stress-free-state variables on structural internal loads and geometric shape. With the knowledge of initial tension in the cables, the tension in any single strands can be determined based on its stress-free-state as well. (Yuan and Qin 2012) For the structural elements fabricated in the factory, the length of elements that are yet to be manufactured can be modified to produce desired overall structural condition by analyzing the effect of length error on the internal load and geometric shape of finished elements (Shi et al. 2010). Designed internal loads and geometric shape can be accurately achieved even when temperature difference between original design and construction site is present (Qin et al. 1995). One-step large-scale cable modification without interference between cables is made possible (Huang 2003) with precision and reduced on-site workload.

\section{Conclusions}

Structural analysis and construction control of staged construction process is a major subject for the modern long-span bridges. This paper introduced the concept of stressfree-state variable of structural elements and briefly deduced the mechanical equilibrium equations and geometric shape governing equations for the staged construction bridge structure utilizing the minimum potential energy theorem. Three basic principles of the stress-free-state theory are drawn as follows,

1) When the stress-free-state variable of a structural element is set, the internal force and deformation of the element are unique at the completion state of the structure regardless of its construction process.

2) The stress-free length of a cable is independent of its external loads, and the change in stress-free length of the cable corresponds to a unique variation of the cable force when the external load is constant.

3) The internal force of a structural element can be independent from its geometric shape within the completion state of a staged construction structure through an active manipulation of stress-free-state variables of the element. 
Stress-free state theory establishes the stage-to-stage and stage-to-completion relationships for the staged construction of bridges, provides a direct and efficient method for theoretical calculations and a flexible and convenient approach for the control of staged construction, and makes parallel construction and auto-filtering of thermal and temporary loading effect possible. The successful applications of the stress-free-state theory in the staged construction controls of nearly one hundred long-span bridges prove that the theory is an efficient and universal method in the structural analysis and construction control for staged construction of bridges.

\section{Acknowledgements}

The authors would thank the colleagues from China Railway Major Bridge Reconnaissance \& Design Institute and Southwest Jiaotong University, for their assistance and discussions in proposing the stress-free-state theory.

\section{Authors' contributions}

Conceptualization, SQ.; Formal analysis, RY, LX and QD; Investigation, SQ, RY, LX, and QD; Supervision, SQ and KW; Writing -original draft, SQ, JQ and KW; Writing - review \& editing, JQ and KW. All authors have read and agreed to the published version of the manuscript.

\section{Funding}

This work was supported financially by the National Natural Science Foundation of China (Grant Nos. 51708455 and 51978578) and the Fundamental Research Fund for Central Universities (A1920502051907-2-001).

\section{Availability of data and materials}

All data, models, and code used during the study appear in the published article.

\section{Competing interests}

Author Shunquan Qin is Editor-in-Chief of Advances in Bridge Engineering.

\section{Author details}

${ }^{1}$ China Railway Major Bridge Reconnaissance \& Design Institute Co., Ltd., Wuhan 430050, China. ${ }^{2}$ Department of Bridge Engineering, Southwest Jiaotong University, Chengdu 610031, China. ${ }^{3}$ Department of Civil and Environmental Engineering, University of California at Los Angeles, Los Angeles 90024, USA.

Received: 30 March 2020 Accepted: 26 May 2020

Published online: 07 July 2020

\section{References}

Dan Q (2017) Alignment controlling theory and application of bridge structure formed in stages based on unstressed state control method. Southwest Jiaotong University, Chengdu

Dan Q, Qin S, Wei K, Xu L (2017a) Equation for geometric shape control of structure formed in stages based on plane beam elements. Bridge Construction 47(4):42-47

Dan Q, Yin Z, Xiang Q, Yuan R, Xu B (2017b) Study on closure technology of double diagonal bar closure section of steel truss girder cable-stayed bridge. Railw Eng 57(10):26-30

Gao B, Huang Y (2003) Construction monitoring and control for Tianjin Tanggu Haihe bridge. Yangtze River 6:59-60

Ge $Y$ (1986) Construction control of cable-stayed bridge - application of filter control theory combined with nonlinear regression analysis in construction of cable-stayed bridge. Tongji University, Shanghai

Ge Y (2003) Analysis and control of staged construction. China Communications Press, Beijing

Huang X (2000) Construction monitoring and control for Wuhan Baishazhou Bridge. In: 2000 bridge academic conference collection of bridge and structural engineering branch of China Highway \& Transportation society. China Communications Press, Beijing, p 409412

Huang X (2003) Construction monitoring and control for 3-pylon cable-stayed spans of Yiling Changjiang River bridge. Bridge Construction 3:22-24

Li Q, Bu Y, Zhang Q (2009) Introduction and application of geometric control in the whole construction process of long-span cable-stayed bridge. Southwest Jiaotong University Press, Chengdu

Li W, Yang G, Yue Q, Wu G (2013) Construction monitoring and control of steel truss girder cable-stayed bridge of Huanggang Changjiang River rail-cum-road bridge. Bridge Construction 43(03):11-17

Liu H, Wen W (2003) Construction monitoring and control for cable-stayed main span of Wuhu Changjiang River bridge. Bridge Construction 03:19-21

Oden JT, Ripperger EA (1986) Elastic structure mechanics. China Architecture \& Building Press, Beijing

Qin S (2003) Control method of stress-free status for erection of cable-stayed bridges. Bridge Constrution 2:31-34

Qin S (2007) Bridge construction control — theory and practice of the stress-free-state method. China Communications Press, Beijing

Qin S (2008a) Key Technology of Tianxingzhou Yangtze River Bridge. In: Nation Structural Engineering Academic Conference

Qin S (2008b) Unstressed state control method for bridges constructed in stages. Bridge Construction 1:8-14

Qin S (2008c) Application of unstressed state control method to calculation for erection of cable-stayed bridge. Bridge Construction 2:13-16 
Qin S (2008d) Techniques for parallel operation of multiple working procedures in construction of cable-stayed bridge. Bridge Construction 3:8-11

Qin S (2009) Unstressed state control method for process control of structure formed by stage. Strategic Study CAE 10:72-78

Qin S, Lin G (1992) Erection calculation of cable-stayed bridge-reviews on the method of reverse disassembly and stressfree-state control. China National Bridge Structure Conference, Wuhan

Qin S, Qin J, Zhang D (2013) Equilibrium equation for spatial frame structure constructed in stages and its applications. IABSE Symp Rep Int Assoc Bridge Structural Eng 101(8):1-8

Qin S, Xie H, Liu X (1995) Erection calculation and monitoring regulation for cable-stayed spans of the second Changjiang River bridge in Wuhan. Bridge Construction 3:41-43

Que S, Yue Q, Yan H (2013) Construction technique for central span and side span closure of steel box girder cable-stayed bridge of Xiamen-Zhangzhou Sea-crossing bridge. World Bridges 41(04):21-25

Shi J, Zhu Y, Li W, Wu Z (2010) Construction monitoring and control of cable-stayed bridge of Wuhan Tianxingzhou Changjiang River rail-cum-road bridge. World Bridges 02:24-28

Sun Y, Xiao H, Xu W (2011) Research of key techniques for construction control of Zhengzhou Huanghe River rail-cum-road bridge. Bridge Construction 02:5-8

Tan K, Yu K (2013) Construction control techniques for four-track curved steel box girder cable-stayed bridge. Bridge Construction 43(04):116-122

Wan S (2012) Application of unstressed state control method in parallel construction of cable-stayed bridge. World Bridge 40(04):59-63

Wu Y (2006) Calculation for construction monitoring of cable-stayed bridge of Lidu Changjiang River bridge in Chongqing. Bridge Construction S2:180-182

Wu Y, Yue Q, Jiang Y (2012) Realization of target geometric shapes of completed bridge of steel box girder cable-stayed bridge based on unstressed state method. Bridge Construction 5:63-68

Wu Y, Yue Q, Zhu L, Huang X (2011) Control measures for closure construction of Jintang primary fairway bridge. Construction Technol 40(03):15-17

Xiang Z (2001) Bridge construction control technology. China Communications Press, Beijing, pp 33-40

Xu L (2016) Analytical theory and method of bridges by two-dimensional staged construction. Southwest Jiaotong University, Chengdu

Xu L, Qin S, Ma R (2013) Equilibrium equation derivation of structures formed by stages based on plane shell element. J Southwest Jiaotong Univ 48(5):857-862

Xu L, Qin S, Yuan R (2015) Equilibrium equation for structure formed in stages based on Timoshenko beam. Bridge Construction 45(5):24-29

Yan H, Yue Q, Que S, Xiao J, Qin Z, Liu H (2013) Construction control method for south main bridge of Xiazhang Seacrossing bridge. World Bridges $41(05): 25-28$

Yu K, Li J (2012) Geometric shape control of cantilever assembled cable-stayed bridge based on unstressed state method. Bridge Construction 3:44-49

Yuan R, Qin S (2012) Application of unstressed state method to construction of steel strand stay cable. Bridge Construction 42(03):75-79

Yuan R, Qin S, Wang F (2014a) Equilibrium equation for geometric nonlinearity of frame structure formed in stage. Bridge Construction 44(2):50-55

Yuan R, Qin S, Wang F (2014b) Equilibrium equation for geometric nonlinearity of structure formed in stages based on plane beam elements. Bridge Construction 44(4):45-49

Yue Q, Yan H, Que S, Liu H, Qin Z (2013) Construction control of long span steel box girder cable-stayed bridge. Bridge Construction 43(04):54-60

Zhang C, Zhang J, Liu C (2015) Study of construction monitoring and control for San'an Yijiang River bridge. World Bridges 43(06):37-41

\section{Publisher's Note}

Springer Nature remains neutral with regard to jurisdictional claims in published maps and institutional affiliations.

\section{Submit your manuscript to a SpringerOpen ${ }^{\circ}$ journal and benefit from:}

- Convenient online submission

- Rigorous peer review

- Open access: articles freely available online

- High visibility within the field

- Retaining the copyright to your article

Submit your next manuscript at $\mathbf{s p r i n g e r o p e n . c o m ~}$ 